\title{
ARTICLE Peripubertal stress-induced heightened aggression: modulation of the glucocorticoid receptor in the central amygdala and normalization by mifepristone treatment
}

\author{
Aurelie Papilloud ${ }^{1}$, Vandana Veenit ${ }^{1,2}$, Stamatina Tzanoulinou ${ }^{1,3}$, Orbicia Riccio ${ }^{1}$, Olivia Zanoletti ${ }^{1}$, Isabelle Guillot de Suduiraut ${ }^{1}$, \\ Jocelyn Grosse ${ }^{1}$ and Carmen Sandi (iD)
}

Despite the enormous negative impact of excessive aggression for individuals and societies, there is a paucity of treatments. Here, using a peripubertal stress model of heightened aggression in rats, we investigated the involvement of the glucocorticoid system and tested the effectiveness of antiglucocorticoid treatment to normalize behavior. We assessed peripubertal stress-induced changes in glucocorticoid (GR) and mineralocorticoid (MR) gene expression in different amygdala nuclei and hippocampus, and report a specific increase in GR mRNA expression in the central amygdala (CeA). Administration of mifepristone (10 mg/kg), a GR antagonist, before stressor exposure at peripuberty prevented the habituation of plasma corticosterone responses observed throughout the stress protocol. This treatment also prevented the increase in aggression and GR expression in the CeA observed in peripubertally stressed rats at adulthood. Viral downregulation of CeA GR expression at adulthood led to reduced aggression. Subsequently, we showed that a brief, 3-day, treatment with mifepristone at adulthood was effective to normalize the abnormal aggression phenotype in peripubertally stressed rats. Our results support a key role for GR actions during peripubertal stress for the long-term programming of heightened aggression. Strikingly, they also support the translational interest of testing the effectiveness of mifepristone treatment to diminish reactive aggression in early adversity-related human psychopathologies.

Neuropsychopharmacology (2019) 44:674-682; https://doi.org/10.1038/s41386-018-0110-0

\section{INTRODUCTION}

Aggression is a behavioral adaptation evolved for its capacity to facilitate an individual's survival or reproduction [1]. Despite the potential advantages of aggression in competitive contests, excessive or inappropriate levels of aggression are frequently maladaptive, incurring on excessive costs for the perpetrating individual and/or its interacting partners [1]. In human societies, excessive aggression is a problem that leads to substantial economic and social costs. Although a great emphasis has been placed in the development of psychosocial programs, their capacity to control pathological aggression is still very limited. Likewise, the results of pharmacological treatments have also been rather unsatisfactory [2], arguably due to the insufficient understanding of the neurobiological mechanisms leading to specific types of aggression [3]. Animal models can help progressing on this knowledge and on the validation of new treatments.

Exposure to adversity during childhood and puberty is a critical contributing factor for the development of pathological aggression and social dysfunctions [4-7]. In humans, this is a period of high sensitivity to develop psychopathological alterations, such as borderline personality disorder [8] that present a high prevalence of reactive aggression [9, 10]. Likewise, exposure to stressful experiences during this period in rats programs for an abnormal pattern of reactive aggression at adulthood [7, 11] that deviates from species-specific norms [12]. Importantly, the same stress protocol, when applied to adult rats, did not induce changes in aggressive behaviors when evaluated after a similar post-stress delay; i.e., two months post-stress [11]. Neuroimaging studies have identified functional alterations in the amygdala in both, aggressive humans with borderline personality disorder $[13,14]$ and aggressive peripubertally stressed rats [11]. Strong evidence implicates the amygdala as a key component in neural circuits involved in emotional processing [15] and on the regulation of social behavior $[16]$ and aggression [17, 18]. Furthermore, features of amygdala structure and function are highly responsive to stress [19].

A system that could be both, involved in the programming of abnormal aggression by peripubertal stress and pharmacologically targetable is the stress-related glucocorticoid system. Glucocorticoids readily cross the blood brain barrier and, through binding to the mineralocorticoid (MR) and glucocorticoid (GR) receptors, they affect a myriad of brain functions via both genomic and nongenomic mechanisms [20, 21]. Importantly, administration of corticosterone-the most prominent glucocorticoid in rodentsduring the peripubertal period mimicked behavioral impairments induced by stress specifically in sociability and aggression, without altering performance in tests for anxiety- and depression-like

\footnotetext{
'Laboratory of Behavioral Genetics, Brain Mind Institute, Ecole Polytechnique Fédérale de Lausanne, Lausanne, Switzerland

Correspondence: Carmen Sandi (carmen.sandi@epfl.ch)

${ }^{2}$ Present address: Departement of Clinical Neuroscience, Karolinska Institutet, Stockholm, Sweden

${ }^{3}$ Present address: Departement of Basic Neurosciences, University of Geneva, 1211 Geneva, Switzerland

These authors contributed equally: Aurelie Papilloud, Vandana Veenit
}

Received: 20 February 2018 Revised: 3 May 2018 Accepted: 28 May 2018

Published online: 4 June 2018 
behaviors [22]. Importantly, GR expression in adulthood can be programmed by early life experiences, a phenomenon particularly studied in the hippocampus [23-25] and recently reported as well for the central amygdala (CeA) [26]. Moreover, increased glucocorticoid levels regulate amygdala activity [27] and GR actions in the CeA have been implicated in the positive modulation of the hypothalamus-pituitary-adrenocortical (HPA) axis [28] and in early life-induced behavioral alterations [26].

Mifepristone, a synthetic steroid with potent GR antagonism [29], is well tolerated in both animals [30] and humans [31]. Mifepristone effectiveness has been shown in clinical trials for psychotic depression [31, 32] and stress-related disorders [33], as well as in preclinical work in rodents [30].

Here, we aimed to assess whether GR expression is modulated by peripubertal stress in the amygdala (i.e., assessing different amygdala nuclei-central, basolateral and medial, and hypothesizing a particular sensitivity of the central nucleus) and whether actions through this receptor are involved in the behavioral programming (i.e., reduced sociability and increased aggression) induced by peripubertal stress exposure. For comparison, we examined as well MR levels and included the hippocampal CA1 region in the analyses. Subsequently, a main goal of this study was to assess whether pharmacological treatment with mifepristone at adulthood is effective in reversing the alterations in sociability and aggression observed in peripubertally stressed animals.

\section{MATERIALS AND METHODS}

Animals

Subjects were the offspring of Wistar Han rats (Charles River Laboratories, France), bred in our animal facility. They were kept on a $12 \mathrm{~h}$ light-dark cycle (lights on at 0700 ). Food and water were available ad libitum. All the procedures were conducted in conformity with Swiss National Institutional Guidelines on Animal Experimentation and approved by a license from the Swiss Cantonal Veterinary Office Committee for Animal Experimentation.

Experimental design

At weaning, male rats from different litters were randomly assigned to control (CTRL) and peripubertal stress (PPS) conditions. They were distributed into home cages in groups of two to three non-siblings. On postnatal day 28 (P28), the peripubertal stress protocol began (see below). At adulthood (P90), animals were handled for three consecutive days before starting the behavioral characterization.

This study was divided into four experiments. In experiment 1, CTRL and PPS rats underwent social preference test at adulthood. One week after the social preference test, animals were split into two experimental manipulations: one group was subjected to a residentintruder test to validate our previous observations regarding increased aggression in PPS rats, whereas the second group was killed and their brains were rapidly extracted for molecular analyses. For the pharmacological blockade of GR, the antagonist mifepristone (RU-486) was injected intraperitoneally $30 \mathrm{~min}$ prior to each peripubertal stress exposure (experiment 2) or on three nonconsecutive days at adulthood (experiment 4), yielding the following four groups: CTRL vehicle, CTRL mifepristone, PPS vehicle and PPS mifepristone. In experiment 3, we targeted GR expression in the CeA, aiming to investigate its role in the modulation of social behaviors. We infused a GR downregulating virus in the CeA of naive adult rats, therefore divided in 2 groups: control scrambled (SCR) and GR-KD rats. In the three last experiments, we evaluated the sociability (social preference test) and aggression (resident-intruder test). All behaviors were video-recorded and manually scored offline by a trained experimenter blind to experimental groups, sometimes assisted by the Observer or Ethovision software (Noldus IT, Netherlands) or inhouse software (Clicker, EPFL, Switzerland). Analysis of gene expression following mifepristone injections at peripuberty was performed at adulthood on an additional cohort of rats.
Peripubertal stress protocol

The peripubertal stress (PPS) protocol is based on exposure to fear-induction procedures, as described in details previously [11]. For more information, see Supplementary Materials and Methods.

Social preference test

The social preference test was adapted from the protocol described by Crawley and colleagues [34] to investigate social affiliation in male mice, following the same conditions previously described [11]. For additional details, see Supplementary Materials and Methods.

Resident-intruder test

The resident-intruder protocol was adapted from ref. [35] and performed following the same conditions as those employed in the previous studies from our laboratory [11, 36]. For further information, see Supplementary Materials and Methods.

\section{Drug treatment}

The glucocorticoid receptor antagonist mifepristone (RU-486, Sigma Aldrich, Switzerland) was dissolved in propylene glycol, thus used as vehicle, at a concentration of $10 \mathrm{mg} / \mathrm{ml}$ and injected intraperitoneally (i.p.) at a dose of $10 \mathrm{mg} / \mathrm{kg}$. Mifepristone or vehicle were injected $30 \mathrm{~min}$ prior to the stress onset on P28-P30, P34, P36, P40 and P42 or on three non-consecutive days, i.e., on P83, P85 and P87 at adulthood. Control groups received vehicle injections at equivalent time as the PPS groups.

\section{Viral downregulation of GR}

We reasoned that $G R$ viral downregulation in the CeA would allow us to address the involvement of GR in the behavioral alterations induced by peripubertal stress (see Results section). However, our previous experience in the lab had indicated a high sensitivity of the stress phenotype to surgery procedures. Therefore, we performed an experiment to test whether infusing peripubertally stressed rats with a scrambled shRNA construct (AAV1/2-U6-SCR shRNA-CAG-EGFP-WPRE-BGH-polyA; SCR) would still allow to observe a pattern of abnormal aggression in stressed animals as compared to control, unstressed, SCR-injected rats. To this end, rats were exposed to the peripubertal stress protocol and, at adulthood, they were subjected to surgeries and infused with the SCR virus (same procedures for control, unstressed rats). Four weeks afterwards, when submitted to the resident-intruder test, we confirmed the interference of surgery procedures with the emergence of an aggressive phenotype in peripubertally stressed rats (Figure S1). Therefore, instead of the planned experiment, we performed an alternative one to investigate the impact of GR knockdown in the CeA in controls.

Thus, at the age of 12 weeks, rats were subjected to surgeries for the viral downregulation of GR in the $\mathrm{CeA}$, wherein an adenoassociated AAV1/2 vector containing an U6-pm-GR3 shRNAterminator-CAG-EGFP-WPRE-BGH-polyA-expression cassette was used. Control animals were injected with the scrambled shRNA construct (see above). All viral constructs used were designed and produced by GeneDetect, New Zealand. For further details, see Supplementary Materials and Methods.

Gene expression analysis, immunofluorescence and immunohistochemistry

For detailed information on gene expression analysis, immunofluorescence and immunohistochemistry, see Supplementary Materials and Methods.

Statistical analysis

Data were analyzed with Student $t$-tests, ANOVAs and repeated measures ANOVAs, as appropriate, using the statistical package GraphPad Prism 5 (GraphPad software Inc, USA). Post hoc analyses were performed with Bonferroni tests. For the $t$-test, if Levene's 
a

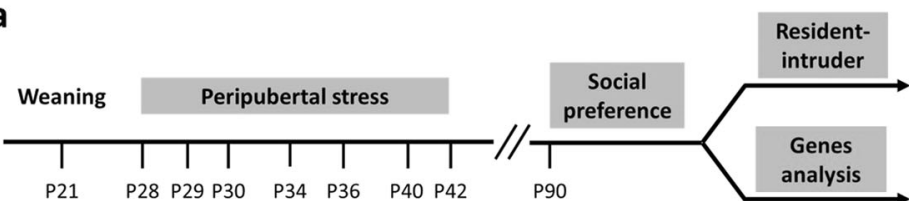

b
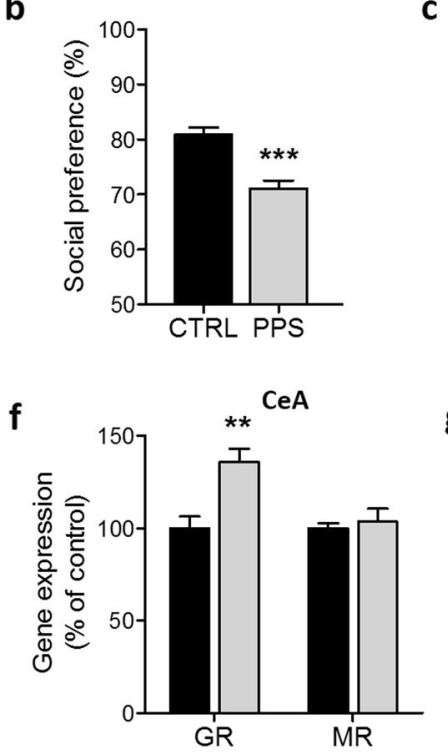
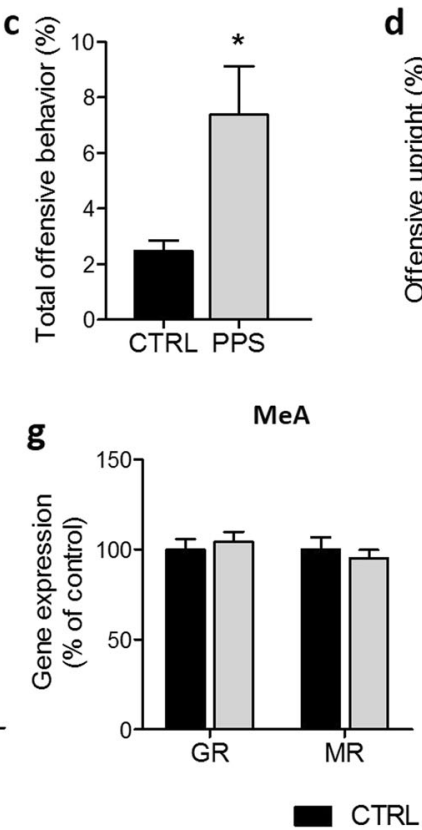

d

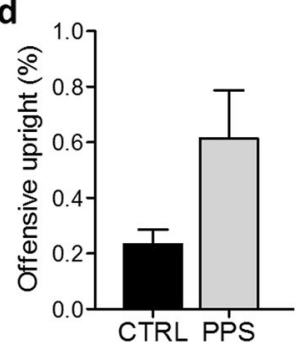

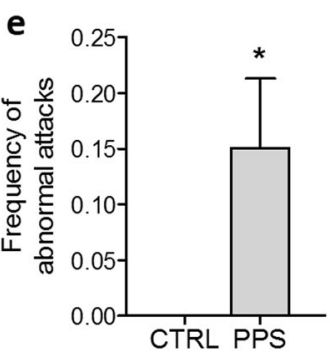
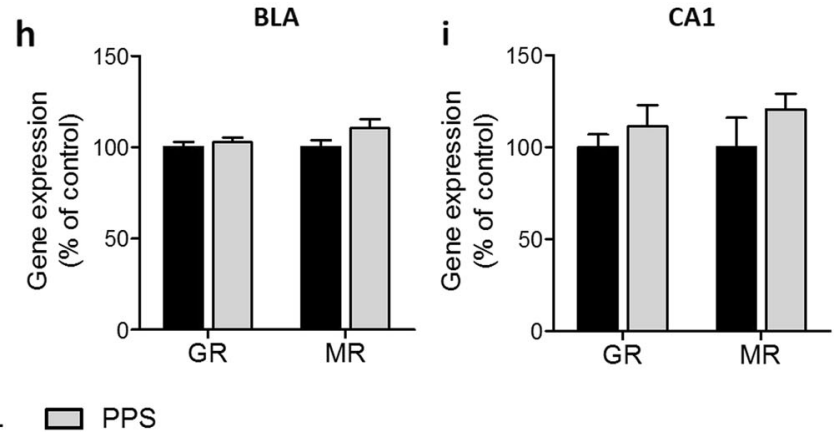

Fig. 1 Effects of peripubertal stress (PPS) on social preference, aggression and GR gene expression. Timeline of the experiment (a). The social preference was lower in stressed rats in comparison to CTRLs (b). PPS animals showed increased percentage of total offensive behavior (c) and frequency of abnormal attacks (e) compared to CTRL rats. The offensive upright percentage did not differ between groups (d). In the central amygdala (CeA), PPS rats showed increased glucocorticoid receptor (GR) mRNA expression without difference in the expression of the mineralocorticoid receptor (MR) (f). No significant change in GR or MR was found in the medial amygdala (MeA) (g), basolateral amygdala $(\mathrm{BLA})(\mathbf{h})$ or CA1 hippocampal region (i). N: social preference: $\mathrm{CTRL}=22, \mathrm{PPS}=25$; aggression: $\mathrm{CTRL}=13, \mathrm{PPS}=16$; gene expression: $\mathrm{CTRL}=$ $8-9$, PPS $=9$. ${ }^{*} p<0.05,{ }^{* *} p<0.01,{ }^{* *} p<0.001$, vs CTRL. Results are expressed as mean \pm SEM

test for equality of variances was significant, equal variance was not assumed and the altered degree of freedom was rounded to the nearest whole number. All bars and error bars represent the mean \pm SEM. Significance was set at $p<0.05$, while the $p$-values were considered tending toward significance when $0.05 \leq p \leq 0.1$. Graphs were created using GraphPad Prism 5.

\section{RESULTS}

Peripubertal stress leads to higher GR expression in the CeA, along with reduced sociability and increased aggression

First, we confirmed our previous findings [11, 36-38] indicating that peripubertal stress leads to reduced sociability and increased aggression when animals are tested at adulthood (Fig. 1a). Indeed, in the sociability test, peripuberty stressed rats showed a reduction in social preference (Fig. $1 b ; t_{15}=3.36, p<0.001$; see Figure S3 for data on the exploration of the juvenile and object). In the resident-intruder test, peripuberty stressed rats exhibited increased total offensive behavior (Fig. $1 c ; t_{26}=2.38, p<0.05$ ) and abnormal attacks (Fig. 1e; $U=63.00, p<0.05$ ), as defined by continuous attacks on intruder exhibiting submissive posture, but did not significantly differ from controls in offensive upright (Fig. 1d; $U=75.50$, n.s.).

Then, GR and MR mRNA levels were measured in different amygdala nuclei and, for comparison, in the CA1 hippocampal area. Peripubertally stressed rats showed increased GR expression in the CeA (Fig. 1f; $t_{15}=3.62, p<0.01$ ). No significant differences were found in the expression of MR (Fig. 1f; $U=32.00$, n.s.) in this brain region, nor in the expression of either $G R$ or $M R$ in the medial amygdala (MeA) (Fig. $1 \mathrm{~g} ; t_{15}=0.55$, n.s. and $U=28.00$, n.s., respectively), basolateral amygdala (BLA) (Fig. $1 \mathrm{~h} ; t_{21}=0.65$, n.s. and $t_{21}=1.60$, n.s., respectively) or CA1 (Fig. $1 \mathrm{i} ; t_{15}=1.43$, n.s. and $U=14.00$, n.s., respectively).

Mifepristone treatment prior to peripubertal stressor exposure prevents stress-induced alterations in sociability, aggression and CeA GR expression

Next, we sought to investigate the implication of GR activation during peripubertal stress exposure on the observed long-term programming of sociability and aggression. The experiment involved pharmacological GR blockade with mifepristone (i.p., $10 \mathrm{mg} / \mathrm{kg}$ ) injected prior to each peripubertal stressor exposure (Fig. 2a). During puberty, mifepristone-injected animals showed higher plasma corticosterone levels following exposure to stressors (Fig. 2b; P28: $F_{(1,16)}=4.41, p<0.05 ; \mathrm{P} 30: F_{(1,16)}=10.41$, $p<0.01$ and P42: $\left.F_{(1,16)}=10.82, p<0.01\right)$. In addition, mifepristone led to a reduction in the corticosterone adaptation to repeated stress exposure (Fig. 2b), a phenomenon that is observed as animals are consecutively exposed to stressors over the peripubertal period (Figure S2).

Animals were tested at adulthood. In the sociability test, stressed rats showed less social preference than CTRLs (Fig. $2 \mathrm{c} ; F_{(1}$, 32) $=9.76, p<0.01)$ but there was no significant stress $\times$ drug treatment interaction $\left(F_{(1,32)}=2.38\right.$, n.s.). A significant stress $\times$ drug treatment interaction was found in the time spent exploring the juvenile during social preference; PPS vehicle rats spent less time exploring the juvenile than CTRL vehicle rats, and this effect was reversed by mifepristone treatment (Figure S4a). 
a

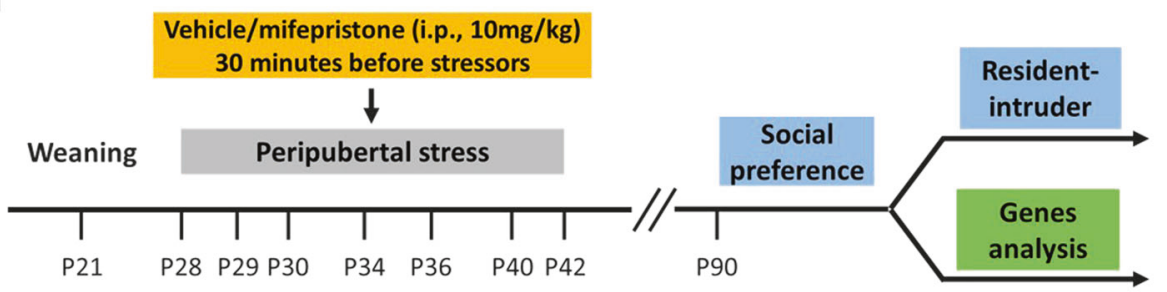

b
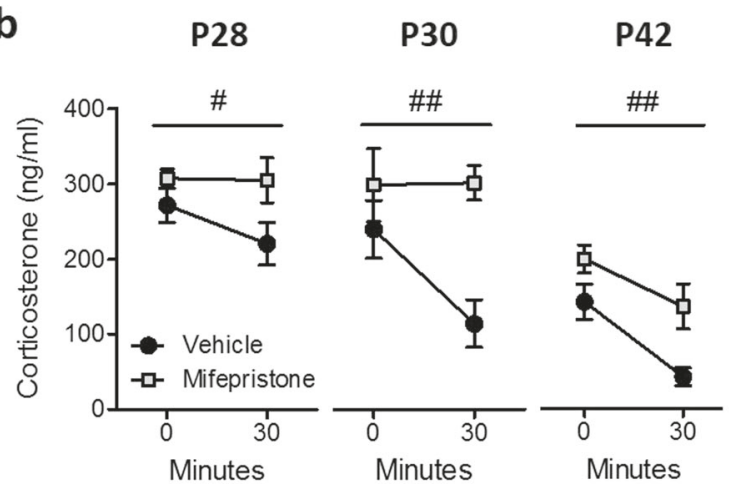

C

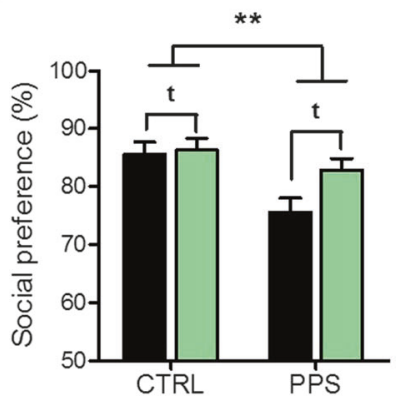

f

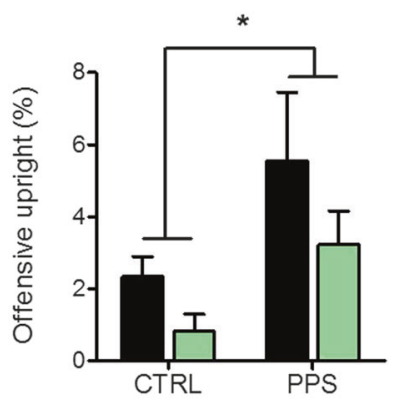

d

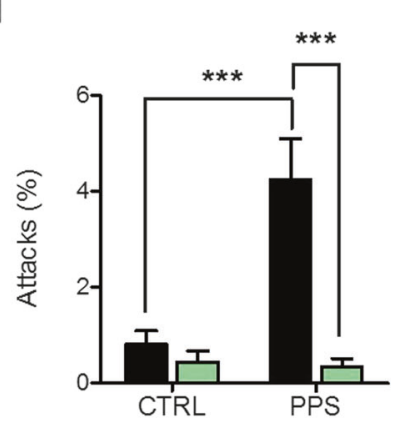

g

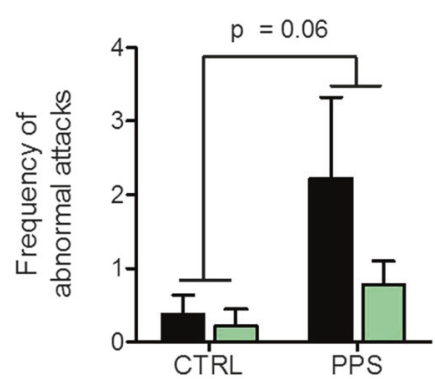

Vehicle e

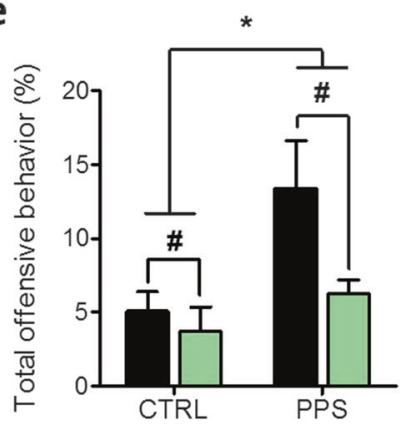

h

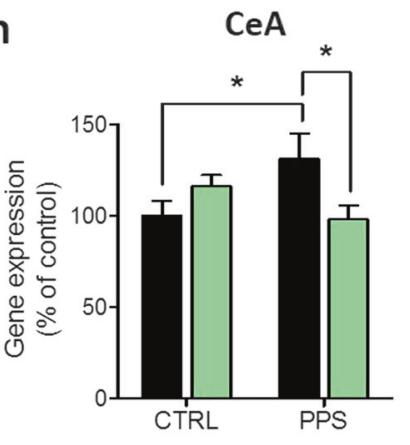

Fig. 2 Effects of mifepristone treatment prior to peripubertal stress (PPS) on corticosterone, social behaviors and GR gene expression in the central amygdala. Timeline of the experiment (a). Increased corticosterone levels were found in mifepristone-treated stressed rats on P28, P30 and P42 compared to PPS rats injected with the vehicle (b). Stressed rats exhibited a decreased social preference, regardless of the drug treatment, whereas a tendency for increased social preference following mifepristone administration was observed in both groups (c). In the resident-intruder test, PPS animals injected with vehicle showed an increased percentage of attacks, which was normalized following mifepristone treatment (d). A main effect of stress and drug treatment were found in the total offensive behavior duration (e). PPS rats showed higher offensive upright duration than CTRLs (f), whereas a trend toward increased abnormal attacks frequency was observed (g). Gene expression analysis revealed an increased glucocorticoid receptor (GR) mRNA level in the central amygdala (CeA) in PPS vehicle rats, which was normalized with mifepristone treatment (h). $N$ : CTRL vehicle $=8-9 ;$ CTRL mifepristone $=8-9$; PPS vehicle $=6-9 ;$ PPS mifepristone $=8-9$. ${ }^{*} p<0.05,{ }^{* *} p<0.01$, vs CTRL vehicle, PPS vehicle or main effect of stress. ${ }^{*} p<0.05,{ }^{\# \#} p<0.01,{ }^{t} p<0.1$ vs vehicle or main effect of drug treatment. Results are expressed as mean \pm SEM 


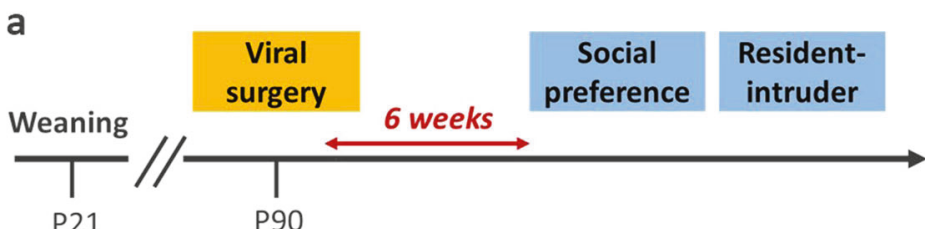

b

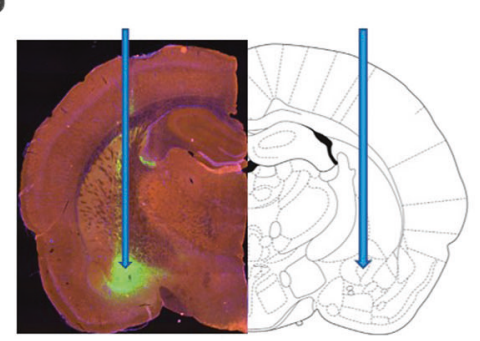

c

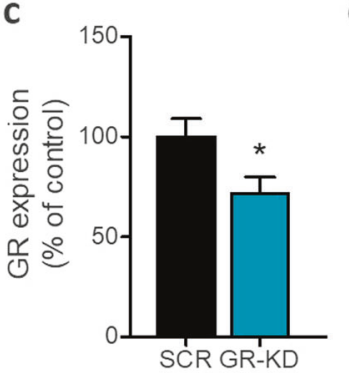

d

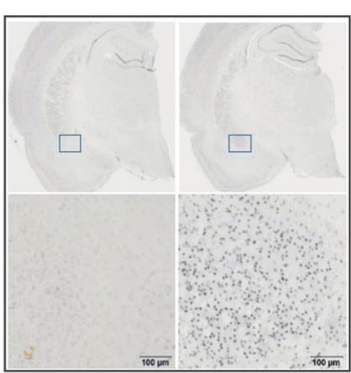

e

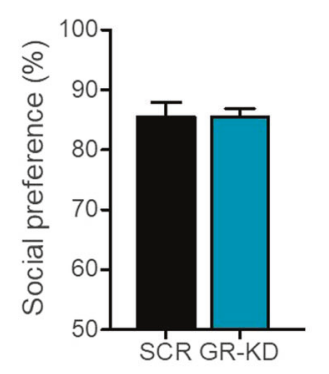

f

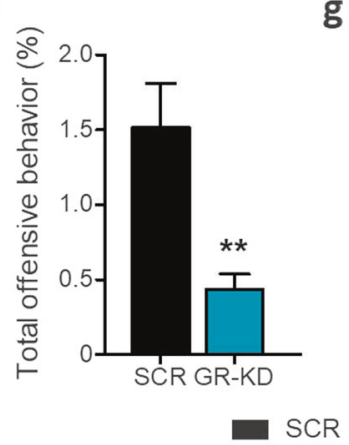

g

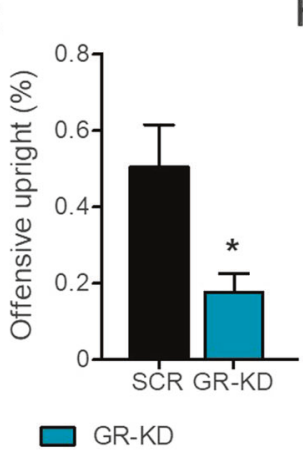

h

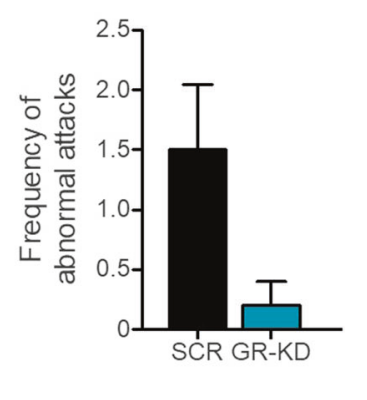

Fig. 3 Glucocorticoid receptor (GR) downregulation and social behaviors. Timeline of the experiment (a). Targeted area in the central amygdala and GFP localization with $\times 4$ magnification of the central amygdala are shown in (b). Analysis of GR expression (mRNA) showed a significant decrease in the central amygdala in rats injected with GR downregulating virus (GR-KD) compared to animals with scrambled (SCR) virus (c). DAB immunohistochemistry confirmed the downregulation of GR protein expression in the central amygdala in GR-KD animals (left panel) compared with SCR rats (right panel) with $\times 40$ magnification (d). The social preference was similar among groups (e). GR-KD rats showed decreased total offensive behavior (f), also observed in the offensive upright (g) duration, as compared to SCR animals. The frequency of abnormal attacks $(\mathbf{h})$ was not found to be significantly different between SCR and GR-KD rats. N: SCR rats $=10-12$ and GR-KD rats $=11-12$, except for resident-intruder data, where GR-KD rats $=6 .{ }^{*} p<0.05,{ }^{* *} p<0.01$, vs SCR. Results are expressed as mean $\pm \mathrm{SEM}$

In the resident-intruder test, ANOVA revealed a significant stress $\times$ treatment interaction for the attack duration (Fig. $2 \mathrm{~d} ; F_{(1,}$ $31)=13.56, p<0.001)$, and post hoc analyses indicated that PPS vehicle rats attacked more than CTRL vehicle animals $(p<0.001)$, whereas PPS mifepristone rats exhibited reduced duration of attack compared to PPS vehicle conspecifics $(p<0.001)$. In addition, there were main effects of stress $\left(F_{(1,31)}=7.11, p<\right.$ $0.05)$ and drug treatment $\left(F_{(1,31)}=4.32, p<0.05\right)$ on total offensive behavior (Fig. 2e). Regarding offensive upright, PPS animals, regardless of the treatment, showed increased percentage compared to CTRL rats (Fig. 2f; $F_{(1,29)}=5.38, p<0.05$ ). Furthermore, a trend toward increased abnormal attacks, as defined by continuous attacks on the intruder exhibiting submissive posture, was observed in PPS animals compared to CTRL conspecifics (Fig. $2 g ; F_{(1,31)}=3.81, p=0.06$ ). A main effect of drug treatment was observed in the latency to first offence (See Figure S4c). Finally, social exploration during the resident-intruder test was similar between groups (Figure S4d).

Interestingly, analyses of gene expression depicted a significant stress $\times$ treatment interaction for GR levels in the CeA (Fig. $2 \mathrm{~h} ; F_{(1}$, 27) $=7.83, p<0.01)$. While PPS vehicle rats showed higher GR expression than CTRL vehicle rats $(p<0.05)$, mifepristone reduced GR expression in PPS animals $(p<0.05)$. No significant stress and/ or drug effects were found in the expression of MR (Figure S5a) in this brain region, nor in the expression of either MR or GR in the
MeA (Figure S5b, c), BLA (Figure S5d, e) or CA1 (Figure S5f, g), with the exception of a significant main effect of mifepristone in reducing GR expression in the CA1 (Figure S5g).

GR downregulation in the $\mathrm{CeA}$ reduces aggressive behavior Findings from the previous experiment show that increased GR expression in the $\mathrm{CeA}$ in peripuberty stressed animals is downregulated by a pre-stress GR blockade treatment that prevents the emergence of several components of the stress-induced behavioral profile. To further understand the link between CeA GR expression and social behaviors, we performed an experiment to assess whether down-regulating GR expression in this brain region in stress-naive animals would produce behavioral effects opposite to those induced by peripubertal stress (see experimental timeline in Fig. 3a).

First, we validated the effectiveness of our AAV-GR downregulation (GR-KD) strategy in reducing GR expression in the CeA (Fig. $3 b-d ; t_{22}=2.09, p<0.05$ for $c$ ). When animals were tested in the sociability test, no effect of treatment was observed in the social preference index (Fig. 3e; $t_{22}=0.02$, n.s.; see Figure S6a for data on the exploration of the juvenile and object). However, in the resident-intruder test, GR-KD rats showed a reduction in total offensive behavior as compared to controls, SCR rats (Fig. 3f; $U=$ $3.00, p<0.01)$. The percentage of offensive upright was also reduced in GR-KD animals (Fig. 3g: $U=8.00, p<0.05$ ). The 


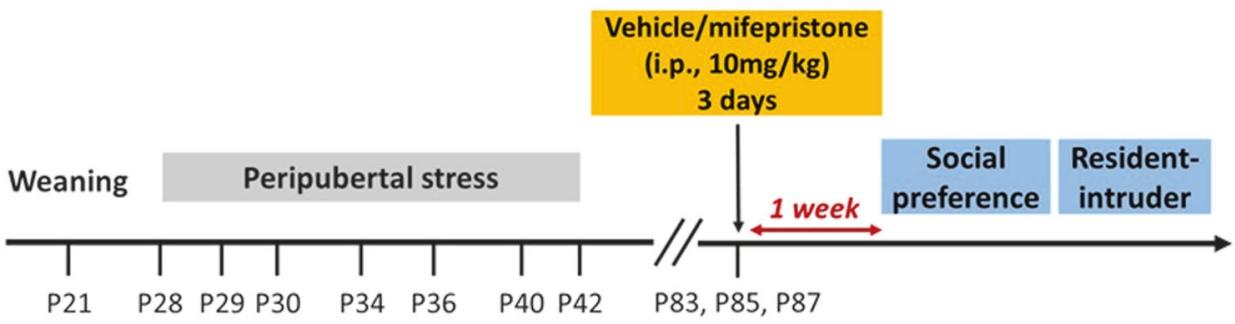

b

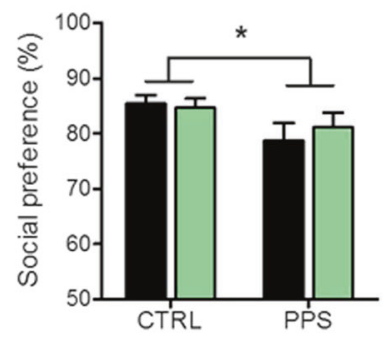

C

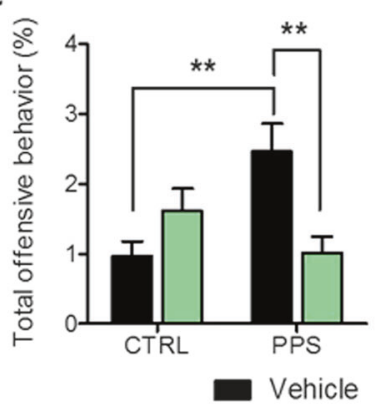

d

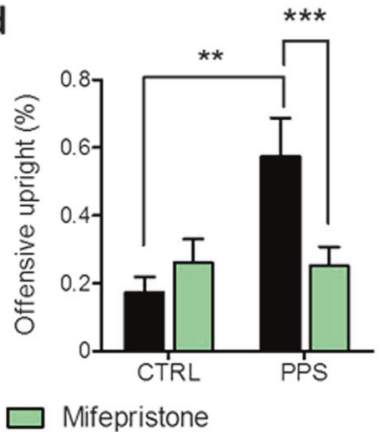

e

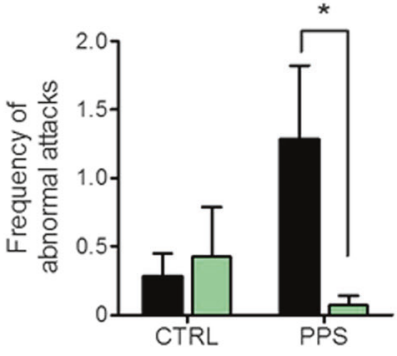

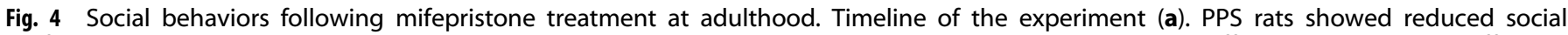
preference ratio compared to CTRLs (b). Stressed animals injected with the vehicle exhibited increased total offensive behavior and offensive

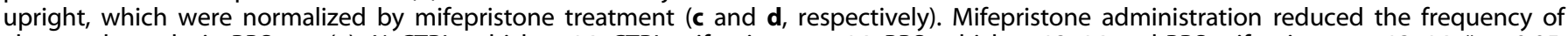
abnormal attacks in PPS rats $(\mathbf{e}) . \quad N$ : CTRL vehicle $=14 ;$ CTRL mifepristone $=14$; PPS vehicle $=12-14$ and PPS mifepristone $=13-14 .{ }^{*} p<0.05$, ${ }^{* *} p<0.01,{ }^{* * *} p<0.001$, vs CTRL vehicle, PPS vehicle or main effect of stress. Results are expressed as mean \pm SEM

frequency of abnormal attacks, as defined by continuous attacks on the intruder that exhibits submissive posture, was not found to be significantly different between the two groups (Fig. 3h; $U=$ 15.00, n.s.). A trend toward increased latency to first offence was also observed in animals injected with the GR downregulating virus, compared to SCR rats (Figure S6b). Finally, the social exploration during the resident-intruder test was similar between groups (Figure S6c).

Mifepristone treatment at adulthood normalizes the abnormal aggression phenotype induced by peripubertal stress

In our previous experiment (see above), mifepristone treatment given every day before daily exposure to the stressors at peripuberty was effective in preventing the emergence of peripubertal stress-induced sociability and aggression alterations. Here, we aimed at investigating the effectiveness of mifepristone treatment when given well after stress had taken place when animals had already reached adulthood. Mifepristone injections (i. p., $10 \mathrm{mg} / \mathrm{kg}$ ) were given on three alternate days (i.e., P83, P85 and P87) and sociability and aggressive behaviors tested 1 week after the end of the treatment (Fig. 4a). In the sociability test, social preference was reduced by peripubertal stress (Fig. $4 \mathrm{~b} ; F_{(1,49)}=$ $5.20, p<0.05$ ), but not modified by mifepristone (Fig. $4 \mathrm{~b} ; F_{(1,49)}=$ 0.04 , n.s.; see Figure S7a, b for data on juvenile and object exploration). In the resident intruder-test, a significant stressxtreatment interaction was found in the percentage of total offensive behavior (Fig. $4 c ; F_{(1,52)}=12.19, p<0.001$ ), offensive upright (Fig. $4 \mathrm{~d} ; F_{(1,52)}=7.38, p<0.01$ ) and frequency of abnormal attacks (Fig. $4 \mathrm{e} ; F_{(1,52)}=4.08, p<0.05$ ). PPS rats injected with vehicle showed increased total offensive behavior and offensive upright $(p<0.01)$ as compared to CTRL vehicle animals, whereas the percentage of those behaviors was reduced in PPS rats treated with mifepristone as compared to PPS vehicle rats $(p<0.01$ or $p<$ 0.001 ). A decreased frequency of abnormal attacks was also found in PPS mifepristone rats compared to stressed animals injected with vehicle (Fig. 4e; $p<0.05$ ). Finally, the latency to first offence (Figure S7c) and the social exploration (Figure S7d) were similar between all groups.

\section{DISCUSSION}

Here, we highlight the GR as a critical mechanism for the regulation of stress-induced aggressive behaviors. We show that GR expression is upregulated in the $\mathrm{CeA}$ in adult rats that show reduced sociability and increased aggression induced by exposure to stress during the peripubertal period [11, 39-41]. Blocking GR actions before stressor exposure at peripuberty prevented the stress-induced emergence of both, the aggressive phenotype and GR modulation. Subsequently, AAV-induced downregulation of GR expression in the $\mathrm{CeA}$ led to reduced aggression while not affecting sociability. Altogether, these findings support the view that GR actions are involved in the long-lasting effects of peripubertal stress on aggression. They also provide the rationale for pharmacologically targeting the GR at adulthood with a mifepristone treatment that successfully normalized stressinduced heightened aggression.

The amygdala is a hub in the brain networks that support social life and its functioning is frequently altered in aggressive individuals $[13,14]$. Rodent studies that allow resolving the engagement of different amygdala nuclei have particularly emphasized the involvement of the $\mathrm{CeA}$ in social behaviors [42] and abnormal aggression [18]. Here, we identify the CeA-but not other amygdala nuclei or the hippocampus-as a critical site for the upregulation of the GR by peripubertal stress. Although the finding of an upregulation seems at odds with previous studies reporting, instead, a CeA downregulation of the GR by increased glucocorticoids [43] or early life stress exposure [26] we found increased GR expression in samples taken at adulthood, but not from samples taken on the aftermath of stress (data not shown). Therefore, the observed increase in CeA GR expression is not an enduring change induced by acute stress/glucocorticoid elevations at peripuberty but, instead, a change that arises later in life. Prepubertal stress is known to modulate responses to stressors experienced at adulthood [44]. Although several explanations could account for our data, evidence suggests the potential involvement of a blunting of corticosterone responsiveness induced by repeated stressor exposure as a possible mechanism involved in this delayed modulation of GR expression [22]. 
Importantly, the fact that animals that develop a heightened aggression pattern are those that show a fast adaptation of their corticosterone responses to repeated stressor exposure during the peripubertal period (i.e., from P28 to P30) is in line with our recent observations in lines of rats genetically selected for their different corticosterone adaptation to that same stressor experience [41]. Specifically, rats with a constitutive fast corticosterone adaptation were the ones that developed a pathological aggression phenotype when exposed to peripubertal stress [41]. Blunted corticosterone responsiveness might be involved, indeed, in the positive regulation of CeA GR expression [45]. Importantly, low corticosterone levels have been linked with the emergence of abnormal forms of aggression and engagement of the $\mathrm{CeA}$ [46, 47]. It is important to note that, in addition to blocking GR activation during stress exposure, we found that pre-stressor exposure mifepristone injections prevented the emergence of the corticosterone habituation to repeated stressor exposure discussed above. This effect is in line with a causal involvement of GR activation in the processes of corticosterone habituation to repeated stress exposure [48].

Although we gathered indirect evidence suggesting a potential association between this observed CeA GR upregulation in adult PPS rats and the reported aggression phenotype, our current data does not allow us to propose a causal link. Correlational evidence for this link is suggested by the fact that mifepristone treatment prevented the enhanced CeA GR expression observed in adult peripubertally stressed rats. In addition, we found a reduction of aggressive behavior induced by AAV-downregulation of the GR in the CeA. However, a causal implication of the PPS-upregulated GR in the CeA in aggressive behavior could only be implied if such AAV-induced GR downregulation in the $\mathrm{CeA}$ would have prevented the emergence of aggression in peripubertally stressed rats. However, due to technical constraints this experiment cannot be performed, as this PPS-induced phenotype is highly sensitive to interference by surgery procedures (see Figure S1).

The efficiency of mifepristone injections pre-stressor exposure at peripuberty in preventing subsequent alterations in aggression suggests the involvement of GR activation during stress exposure for the emergence of these effects. These results are aligned with evidence in hamsters showing that an acceleration of the transition from play fighting to adult aggression induced by enhanced glucocorticoid levels during puberty is prevented by mifepristone [49]. They are also in line with our previous work showing increased aggressive behavior induced by a corticosterone regime of injections that corresponded with the peripubertal stress schedule [22]. Note, however, that we do not find signs of abnormal aggression elicited by the peripubertal corticosterone treatment when reanalyzing our early data from [22], suggesting that other mechanisms, in addition to glucocorticoids, are contributing to the long-term programming of abnormal aggression induced by peripubertal stress.

Given our findings on the CeA discussed above, we should note that peripheral mifepristone treatment is known to affect $\mathrm{CeA}$ activation by stress [50], and mifepristone actions specifically in the $\mathrm{CeA}$ were previously shown to reverse stress-induced behavioral adaptations [51]. Interestingly, enhancing glucocorticoid levels either peripherally [52] or in the CeA [53] was shown to increase expression levels of corticotropin-releasing factor (CRF) in the CeA. We previously showed that peripubertal stress enhances expression of the CRF receptor 1 (CRFR1) in the CeA, and treatment with a CRFR1 antagonist in the week following peripuberty stress exposure prevented the occurrence of psychopathological behaviors at adulthood (note, however, that aggressive behavior was not tested in the study) [40]. Given that stressinduced release of $\mathrm{CRH}$ was shown to be prevented by mifepristone [54], these body of data support the hypothesis that the modulation of the CRF system by CeA GR actions might be implicated in the social dysfunctions induced by peripubertal stress. However, we should cautiously note that we cannot imply the involvement, at least not exclusively, of CeA GR in the effects of peripheral mifepristone treatment. Several other brain areas could also mediate corticosterone actions in the context of aggression $[18,55]$. Notably, several hypothalamic nuclei, such as the hypothalamic attack area, have been implicated in the control of aggression and can be responsive to activation by corticosterone $[55,56]$.

Finally, we report that mifepristone treatment at adulthood, uncoupled from the stress experience, normalized the abnormal aggression phenotype in peripubertally stressed rats. Previous work in rodents had reported the effectiveness of brief mifepristone treatments to reverse behavioral alterations induce by former stress experiences. Thus, mifepristone, given during the last 4 days of a chronic stress protocol, was shown to reverse stress-induced alterations in hippocampal structure and function [57]. In another study, mifepristone administered $1 \mathrm{~h}$ before testing, reversed maternal separation stress-induced behavioral dysfunctions in depression-related and spatial memory tests [58]. More recently, a series of studies showed that a 3-day mifepristone treatment, uncoupled to both previous stress and behavioral testing, could reverse long-term effects of stress. Specifically, a 3-day mifepristone treatment during the prepuberty period could restore the long-term negative impact of early maternal deprivation on fear memories $[30,59]$. Here, we present striking evidence for a full reversal of the heightened aggression phenotype induced by peripubertal stress exposure by a 3-day mifepristone treatment that was uncoupled from both exposure to early adversity and the resident-intruder test for aggression, which took place 2 weeks after drug treatment. However, the same treatment did not reverse the reduced sociability induced by peripubertal stress. Further studies are needed to interrogate the mechanisms whereby mifepristone normalized aggression. Importantly, a link between fear and aggression [46] has been proposed. The fact that mifepristone was previously shown to normalize enhanced fear learning induced by early life stress [30, 59] suggests that a reduction in the emotional reactivity towards intruder conspecifics might have operated to reduce aggression in mifepristone-treated rats. Importantly, mifepristone is already used as treatment for diverse medical conditions in humans, including Cushing's syndrome [60] and psychotic depression [32].

Altogether, our results show that GR expression in the CeA is susceptible to modulation in opposite ways by peripubertal stress and GR antagonism, two treatments that have as well opposite effects in adult aggression. From a translational perspective, the success of mifepristone treatment given at adulthood supports the interest of testing anti-glucocorticoid treatments to diminish reactive aggression in early adversity-related human psychopathologies [61].

\section{ACKNOWLEDGEMENTS}

We thank Jocelyn Grosse Elodie Schranz, Arthur Barakat, Céline Fournier, Damien Huzard and Sophie Walker for their excellent assistance in the performance of the stress protocol. This project has been supported by grants from the Swiss National Science Foundation (31003A-152614; and NCCR Synapsy, Grant No. 51NF40-158776), European Union's Seventh Framework Program for research, technological development and demonstration under Grant Agreement No. 603016 (MATRICS), and intramural funding from the EPFL to CS.

\section{ADDITIONAL INFORMATION}

Supplementary Information accompanies this paper at (https://doi.org/10.1038/ s41386-018-0110-0)

Competing interests: The authors declare no competing interests.

Publisher's note: Springer Nature remains neutral with regard to jurisdictional claims in published maps and institutional affiliations. 


\section{REFERENCES}

1. Takahashi, A, and Miczek, KA. Neurogenetics of aggressive behavior: studies in rodents. Curr Top Behav Neurosci. 2014;17:3-44.

2. Glannon W. Intervening in the psychopath's brain. Theor Med Bioeth. 2014;35:43-57.

3. Dadds MR, Rhodes T. Aggression in young children with concurrent callousunemotional traits: can the neurosciences inform progress and innovation in treatment approaches? Philos Trans R Soc Lond B Biol Sci. 2008;363:2567-76.

4. Haller J, Harold G, Sandi C, Neumann ID. Effects of adverse early-life events on aggression and anti-social behaviours in animals and humans. J Neuroendocrinol. 2014;26:724-38.

5. Provençal N, Booij L, Tremblay RE. The developmental origins of chronic physical aggression: biological pathways triggered by early life adversity. J Exp Biol. 2015;218:123-33.

6. Sandi C, Haller J. Stress and the social brain: behavioural effects and neurobiological mechanisms. Nat Rev Neurosci. 2015;16:290-304.

7. Tzanoulinou S, Sandi C. The programming of the social brain by stress during childhood and adolescence: from rodents to humans. Curr Top Behav Neurosci. 2017;30:411-29.

8. Newnham EA, Janca A. Childhood adversity and borderline personality disorder: a focus on adolescence. Curr Opin Psychiatry. 2014;27:68-72.

9. McCloskey MS, New AS, Siever LJ, Goodman M, Koenigsberg HW, Flory JD, et al. Evaluation of behavioral impulsivity and aggression tasks as endophenotypes for borderline personality disorder. J Psychiatr Res. 2009;43:1036-48.

10. Coccaro EF, Lee R, McCloskey M, Csernansky JG, Wang L. Morphometric analysis of amygdla and hippocampus shape in impulsively aggressive and healthy control subjects. J Psychiatr Res. 2015;69:80-86.

11. Márquez $\mathrm{C}$, Poirier $\mathrm{GL}$, Cordero $\mathrm{MI}$, Larsen $\mathrm{MH}$, Groner $\mathrm{A}$, Marquis J, et al. Peripuberty stress leads to abnormal aggression, altered amygdala and orbitofrontal reactivity and increased prefrontal MAOA gene expression. Transl Psychiatry. 2013;3:e216.

12. Haller J. Studies into abnormal aggression in humans and rodents: methodological and translational aspects. Neurosci Biobehav Rev. 2017;76:77-86.

13. Rosell DR, Siever LJ. The neurobiology of aggression and violence. CNS Spectr. 2015;20:254-79.

14. Coccaro EF, McCloskey MS, Fitzgerald DA, Phan KL. Amygdala and orbitofrontal reactivity to social threat in individuals with impulsive aggression. Biol Psychiatry. 2007;62:168-78.

15. Phelps EA, LeDoux JE. Contributions of the amygdala to emotion processing: from animal models to human behavior. Neuron. 2005;48:175-87.

16. Adolphs R. What does the amygdala contribute to social cognition? Ann N Y Acad Sci. 2010;1191:42-61.

17. Glenn AL, Raine A. Neurocriminology: implications for the punishment, prediction and prevention of criminal behaviour. Nat Rev Neurosci. 2014;15:54-63.

18. Haller J. The role of central and medial amygdala in normal and abnormal aggression: a review of classical approaches. Neurosci Biobehav Rev. 2018;85:34-43.

19. Roozendaal B, McEwen BS, Chattarji S. Stress, memory and the amygdala. Nat Rev Neurosci. 2009;10:423-33.

20. de Kloet ER, Karst H, Joëls M. Corticosteroid hormones in the central stress response: Quick-and-slow. Front Neuroendocrinol. 2008;29:268-72.

21. Joëls $M$, Pasricha $N$, Karst $H$. The interplay between rapid and slow corticosteroid actions in brain. Eur J Pharmacol. 2013;719:44-52.

22. Veenit V, Cordero MI, Tzanoulinou S, Sandi C. Increased corticosterone in peripubertal rats leads to long-lasting alterations in social exploration and aggression. Front Behav Neurosci. 2013;7:26.

23. Enthoven L, Schmidt MV, Cheung YH, van der Mark MH, de Kloet ER, Oitzl MS. Ontogeny of the HPA axis of the CD1 mouse following 24h maternal deprivation at pnd 3. Int J Dev Neurosci. 2010;28:217-24.

24. Santarelli S, Zimmermann C, Kalideris G, Lesuis SL, Arloth J, Uribe A, et al. An adverse early life environment can enhance stress resilience in adulthood. Psychoneuroendocrinology. 2017;78:213-21.

25. Sutanto $W$, Rosenfeld $P$, de Kloet ER, Levine $S$. Long-term effects of neonatal maternal deprivation and ACTH on hippocampal mineralocorticoid and glucocorticoid receptors. Brain Res Dev Brain Res. 1996;92:156-63.

26. Arnett MG, Pan MS, Doak W, Cyr PEP, Muglia LM, Muglia LJ. The role of glucocorticoid receptor-dependent activity in the amygdala central nucleus and reversibility of early-life stress programmed behavior. Transl Psychiatry. 2015;5:e542.

27. Henckens MJAG, van Wingen GA, Joëls M, Fernández G. Corticosteroid induced decoupling of the amygdala in men. Cereb Cortex. 2012;22:2336-45.

28. Ulrich-Lai YM, Herman JP. Neural regulation of endocrine and autonomic stress responses. Nat Rev Neurosci. 2009:10:397-409.

29. van Haarst AD, Oitzl MS, Workel JO, de Kloet ER. Chronic brain glucocorticoid receptor blockade enhances the rise in circadian and stress-induced pituitaryadrenal activity. Endocrinology. 1996;137:4935-43.
30. Loi M, Sarabdjitsingh RA, Tsouli A, Trinh S, Arp M, Krugers HJ, Karst $H$, et al Transient prepubertal mifepristone treatment normalizes deficits in contextual memory and neuronal activity of adult male rats exposed to maternal deprivation. eNeuro. 2017:4:0253-17.

31. Blasey CM, Block TS, Belanoff JK, Roe RL. Efficacy and safety of mifepristone for the treatment of psychotic depression. J Clin Psychopharmacol. 2011;31:436-40.

32. Flores BH, Kenna H, Keller J, Solvason HB, Schatzberg AF. Clinical and biological effects of mifepristone treatment for psychotic depression. Neuropsychopharmacology. 2006;31:628-36.

33. Golier JA, Caramanica K, Michaelides AC, Makotkine I, Schmeidler J, Harvey PD, et al. A randomized, double-blind, placebo-controlled, crossover trial of mifepristone in Gulf War veterans with chronic multisymptom illness. Psychoneuroendocrinology. 2016;64:22-30.

34. Moy SS, Nadler JJ, Perez A, Barbaro RP, Johns JM, Magnuson TR, Piven J, et al. Sociability and preference for social novelty in five inbred strains: an approach to assess autistic-like behavior in mice. Genes Brain Behav. 2004;3:287-302.

35. Veenema AH, Blume A, Niederle D, Buwalda B, Neumann ID. Effects of early life stress on adult male aggression and hypothalamic vasopressin and serotonin. Eur J Neurosci. 2006;24:1711-20.

36. Cordero Ml, Ansermet F, Sandi C. Long-term programming of enhanced aggression by peripuberty stress in female rats. Psychoneuroendocrinology. 2013;38:2758-69.

37. Poirier GL, Imamura N, Zanoletti O, Sandi C. Social deficits induced by peripubertal stress in rats are reversed by resveratrol. J Psychiatr Res. 2014; 57:157-64.

38. Tzanoulinou S, Riccio O, de Boer MW, Sandi C. Peripubertal stress-induced behavioral changes are associated with altered expression of genes involved in excitation and inhibition in the amygdala. Transl Psychiatry. 2014; 4:e410.

39. Cordero MI, Just N, Poirier GL, Sandi C. Effects of paternal and peripubertal stress on aggression, anxiety, and metabolic alterations in the lateral septum. Eur Neuropsychopharmacol. 2016;26:357-67.

40. Veenit V, Riccio O, Sandi C. CRHR1 links peripuberty stress with deficits in social and stress-coping behaviors. J Psychiatr Res. 2014;53:1-7.

41. Walker, SE, and Sandi, C. Long-term programing of psychopathology-like behaviors in male rats by peripubertal stress depends on individual's glucocorticoid responsiveness to stress. Stress 2018 Feb 7:1-10.

42. Calcagnoli F, Stubbendorff C, Meyer N, De Boer SF, Althaus M, Koolhaas JM Oxytocin microinjected into the central amygdaloid nuclei exerts anti-aggressive effects in male rats. Neuropharmacology. 2015;90:74-81.

43. Tran L, Greenwood-Van Meerveld B. Altered expression of glucocorticoid receptor and corticotropin-releasing factor in the central amygdala in response to elevated corticosterone. Behav Brain Res. 2012;234:380-5.

44. Tsoory M, Richter-Levin G. Learning under stress in the adult rat is differentially affected by "juvenile" or "adolescent" stress. Int J Neuropsychopharmacol. 2006;9:713-28.

45. Herman, JP, McKlveen, JM, Ghosal, S, Kopp, B, Wulsin, A, Makinson, R, Scheimann, $\mathrm{J}$, et al. Regulation of the hypothalamic-pituitary-adrenocortical stress response. Comprehens Physiol. 2016;6:603-21.

46. Halász J, Liposits Z, Kruk MR, Haller J. Neural background of glucocorticoid dysfunction-induced abnormal aggression in rats: involvement of fear- and stress-related structures. Eur J Neurosci. 2002;15:561-9.

47. Tulogdi A, Toth M, Halasz J, Mikics E, Fuzesi T, Haller J. Brain mechanisms involved in predatory aggression are activated in a laboratory model of violent intraspecific aggression. Eur J Neurosci. 2010;32:1744-53.

48. Pace TW, Cole MA, Ward G, Kalman BA, Spencer RL. Acute exposure to a nove stressor further reduces the habituated corticosterone response to restraint in rats. Stress. 2001:4:319-31.

49. Wommack JC, Delville Y. Cortisol controls the pubertal development of agonistic behavior in male golden hamsters via type II corticosteroid receptors. Horm Behav. 2007;51:306-12.

50. Wulsin AC, Herman JP, Solomon MB. Mifepristone decreases depression-like behavior and modulates neuroendocrine and central hypothalamic-pituitaryadrenocortical axis responsiveness to stress. Psychoneuroendocrinology. 2010;35:1100-12.

51. Simms JA, Haass-Koffler CL, Bito-Onon J, Li R, Bartlett SE. Mifepristone in the central nucleus of the amygdala reduces yohimbine stress-induced reinstatement of ethanol-seeking. Neuropsychopharmacology. 2012;37:906-18.

52. Schulkin J, Morgan MA, Rosen JB. A neuroendocrine mechanism for sustaining fear. Trends Neurosci. 2005;28:629-35.

53. Shepard JD, Barron KW, Myers DA. Corticosterone delivery to the amygdala increases corticotropin-releasing factor mRNA in the central amygdaloid nucleus and anxiety-like behavior. Brain Res. 2000;861:288-95.

54. Cook CJ. Glucocorticoid feedback increases the sensitivity of the limbic system to stress. Physiol Behav. 2002;75:455-64. 
55. Haller J. The glucocorticoid/aggression relationship in animals and humans: an analysis sensitive to behavioral characteristics, glucocorticoid secretion patterns, and neural mechanisms. Curr Top Behav Neurosci. 2014;17:73-109.

56. Kruk MR, Haller J, Meelis W, de Kloet ER. Mineralocorticoid receptor blockade during a rat's first violent encounter inhibits its subsequent propensity for violence. Behav Neurosci. 2013;127:505-14.

57. Krugers HJ, Goltstein PM, Van Der Linden S, Joëls M. Blockade of glucocorticoid receptors rapidly restores hippocampal CA1 synaptic plasticity after exposure to chronic stress. Eur J Neurosci. 2006;23:3051-5.

58. Aisa B, Tordera R, Lasheras B, Del Río J, Ramírez MJ. Cognitive impairment associated to HPA axis hyperactivity after maternal separation in rats. Psychoneuroendocrinology. 2007;32:256-66.
59. Arp JM, ter Horst JP, Loi M, den Blaauwen J, Bangert E, Fernández G, et al. Blocking glucocorticoid receptors at adolescent age prevents enhanced freezing between repeated cue-exposures after conditioned fear in adult mice raised under chronic early life stress. Neurobiol Learn Mem. 2016;133: 30-38.

60. Fleseriu M, Biller BMK, Findling JW, Molitch ME, Schteingart DE, Gross C. Mifepristone, a glucocorticoid receptor antagonist, produces clinical and metabolic benefits in patients with Cushing's syndrome. J Clin Endocrinol Metab. 2012;97:2039-49.

61. Kruk MR, Halász J, Meelis W, Haller J. Fast positive feedback between the adrenocortical stress response and a brain mechanism involved in aggressive behavior. Behav Neurosci. 2004;118:1062-70. 